\title{
MODELAGEM DE TORQUE PARA MOTORES COM TECNOLOGIA FLEX
}

\author{
Marcos Henrique C. Silva ${ }^{1}$, Armando Antônio M. Laganá ${ }^{2}$ \\ ${ }^{1,2}$ Escola Politécnica da Universidade de São Paulo \\ ${ }^{2}$ Fatec Santo André
}

E-mails: marcoshencarsil@gmail.com, armandolagana@terra.com.br

\section{RESUMO}

Neste artigo é apresentado um modelo de torque para motores com tecnologia FLEX. O objetivo é fornecer uma formulação matemática, baseada em métodos de identificação experimental e análise teórica, que prediz o torque produzido pelo motor a partir da geometria do motor e de variáveis temporais conhecidas, como o poder calorífico inferior da mistura injetada, a massa de combustível injetada, a velocidade angular do virabrequim, a relação ar/combustível, a pressão no coletor de admissão e o ângulo de ignição. Será analisado também como a composição etanol/gasolina influencia nestes fatores, de forma a expandir o modelo para o uso em motores FLEX. Para esta análise, empregar-se-á correlação dentre as variáveis, variância das medidas, técnicas de identificação e estudo físico-químico dos efeitos do uso de composição variada.

\section{INTRODUÇÃO}

A formulação para combustível FLEX da eficiência térmica, acompanhada da formulação do torque efetivo, segue abaixo:

$$
\begin{gathered}
e_{c o r}(.)=e_{\omega_{e}}\left(\omega_{e}, \Upsilon\right) \cdot e_{\lambda}(\lambda, \Upsilon) \cdot e_{\zeta}(\zeta, \Upsilon) \cdot e_{e g r}\left(x_{e g r}\right)+p_{m e 0 g}(0) \cdot K \\
\cdot \frac{\lambda \cdot \sigma_{0} \cdot V_{d}}{H_{l} \cdot m_{\beta, \max }\left(\omega_{e}\right)} \\
T_{e}=e_{c o r}(\cdot) \cdot m_{\varphi} \cdot H_{l}-\frac{V_{d}}{4 \cdot \pi} \cdot\left[p_{m e o g}(0)+p_{m e 0 f}(.)\right]
\end{gathered}
$$

Aonde, 
$e_{c o r}($.$) : Eficiência térmica; \omega_{e}$ : Velocidade do motor; $e_{\omega_{e}}\left(\omega_{e}, \Upsilon\right)$ : Fator da eficiência térmica dependente da velocidade do motor; $e_{\lambda}(\lambda, \Upsilon)$ : Fator da eficiência térmica dependente da relação equivalente ar/combustível; $\zeta$ : Ângulo de ignição; $e_{\zeta}(\zeta, \Upsilon)$ : Fator da eficiência térmica dependente do ângulo de ignição; $x_{e g r}$ : Taxa de recirculação de gases (pode ser mássica ou molar); $e_{e g r}\left(x_{e g r}\right)$ : Fator da eficiência térmica dependente da taxa de recirculação de gases; $p_{m e o g}(0)$ : Perdas devido ao atrito do fluxo gasoso no cilindro e no sistema de exaustão; $K$ : Constante; $\lambda$ : Relação equivalente ar/combustível; $\sigma_{0}$ : Relação estequiométrica ar/combustível; $V_{d}$ : Volume de deslocamento; $H_{l}$ : Poder calorífico inferior da mistura de combustível utilizada; $m_{\beta}$ : Valor mássico da mistura de ar com gases queimados recirculados admitida pelo cilindro em um ciclo determinado; $m_{\beta, \max }\left(\omega_{e}\right)$ : Máximo valor de $m_{\beta}$ possível de se obter em determinada velocidade do motor; $T_{e}$ : Valor médio de torque produzido em um ciclo determinado; $m_{\varphi}$ : Valor mássico da mistura de combustível admitida pelo cilindro em um ciclo determinado; $p_{m e 0 f}($.): Engloba as perdas devido às fricções das partes móveis mecânicas do motor e as perdas devido ao funcionamento de dispositivos auxiliares, como as bombas de água e óleo, o ar-condicionado, a ventoinha, dentre outros.

$O$ termo $\Upsilon$ refere-se a um fator normalizado (que vai de 0 à 1) que varia linearmente segundo a composição. Para composições de gasolina tipo A com etanol anidro (escala EX) será usado $\Upsilon=0$ para gasolina tipo $A$ e $Y=1$ para etanol anidro. Para composições de gasolina tipo C (E27) com etanol hidratado (escala HX, vide [1]) será usado $\Upsilon=0$ para gasolina tipo $C$ e $\Upsilon=1$ para etanol hidratado.

Neste artigo discorreremos sobre como fazer a identificação das fórmulas acima.

Em uma abordagem inicial, recomenda-se considerar $K=0$ e fazer a identificação dos fatores $e_{\omega_{e}}\left(\omega_{e}, \Upsilon\right), e_{\zeta}(\zeta, \Upsilon)$ e $e_{e g r}\left(x_{e g r}\right)$.

Como pretendemos deixar os fatores $e_{\zeta}(\zeta, \Upsilon), e_{e g r}\left(x_{e g r}\right)$ e $e_{\lambda}(\lambda, \Upsilon)$ normalizados (ou seja, variando de 0 à 1 ), temos que computar em $e_{\omega_{e}}\left(\omega_{e}, \Upsilon\right)$ as perdas devido às 
limitações de conversão de energia do ciclo termodinâmico e às transferências de calor incontornáveis. Logo, o valor de $e_{\omega_{e}}\left(\omega_{e}, \Upsilon\right)$ será consideravelmente menor que 1 , sendo idealmente o valor da eficiência de um ciclo Otto ideal.

Recomenda-se, a seguir, identificar $e_{\lambda}(\lambda, \Upsilon)$ conjuntamente com $K$.

Este artigo é desenvolvido para motores a combustão interna de 4 tempos, com injeção indireta na porta, naturalmente aspirados e com combustão por centelha.

\section{Fator $e_{\omega_{e}}\left(\omega_{e}, \mathbf{Y}\right)$}

Para identificar este fator, calcularemos o torque que seria obtido se tivéssemos total conversão da energia de combustão disponível em energia efetiva, conforme equação (3) abaixo:

$$
T_{\text {NO-LOSS }}=\frac{H_{l} \cdot m_{\varphi}}{4 \pi}
$$

A seguir, compararemos o valor de $T_{N O-L O S S}$ com o valor obtido através da curva de torque e obteremos $e_{\omega_{e}}\left(\omega_{e}, \Upsilon\right)$. Uma forma de fazer isso é dividir a ordenada $T_{e, c u r v a}$ da curva de torque por $T_{N O-L O S S}$, fazendo:

$$
e_{\omega_{e}}\left(\omega_{e}, 0\right)=\frac{T_{e, \text { curva }}\left(\omega_{e}\right)}{T_{N O-L O S S}}
$$

Deve-se fazer o mesmo para composições representativas (por exemplo, para escala EX, recomenda-se usar E0, E25, E50, E75 e E100). Ao terminar este levantamento, deve-se atentar como pode usar fatores de correção para aproximar uma curva da outra, pois as curvas costumam ter a mesma morfologia, porém magnitudes diferentes. Logo, por exemplo, para uma curva que obedeça este comportamento, mas que tenha espaçamento diferente para $\omega_{e} \leq \omega_{O P T}$ e $\omega_{e} \geq \omega_{O P T}$, sendo $\omega_{O P T}$ a velocidade do motor na qual ocorre torque máximo, teríamos: 


$$
e_{\omega_{e}}\left(\omega_{e}, \Upsilon\right)= \begin{cases}f(\Upsilon) \cdot e_{\omega_{e}}\left(\omega_{e}, 0\right), & \operatorname{para} \omega_{e} \leq \omega_{O P T} \\ g(\Upsilon) \cdot e_{\omega_{e}}\left(\omega_{e}, 0\right) & \text { para } \omega_{e} \geq \omega_{O P T}\end{cases}
$$

Aonde $f(\Upsilon)$ e $g(\Upsilon)$ exercem aqui o papel de fatores de correção. São funções afim (têm o formato $a \cdot \Upsilon+$ b) que devem ser obtidas através de métodos de identificação.

Um levantamento que obedece a equação (5) segue reproduzido na figura 1 abaixo. Repare que, das equações (1) e (2), temos a relação (6).

$$
\frac{\partial T_{e}}{\partial \omega_{e}} \approx K_{2} \cdot \frac{\partial e_{\omega_{e}}\left(\omega_{e}, \Upsilon\right)}{\partial \omega_{e}}
$$

Logo, podemos usar as curvas de torque para ter uma visão qualitativa de como o fator $e_{\omega_{e}}\left(\omega_{e}, \Upsilon\right)$ varia com a velocidade do motor.

Figura 1: Curvas características do motor para composição variada. Uso de composição EX num motor de ignição por centelha.

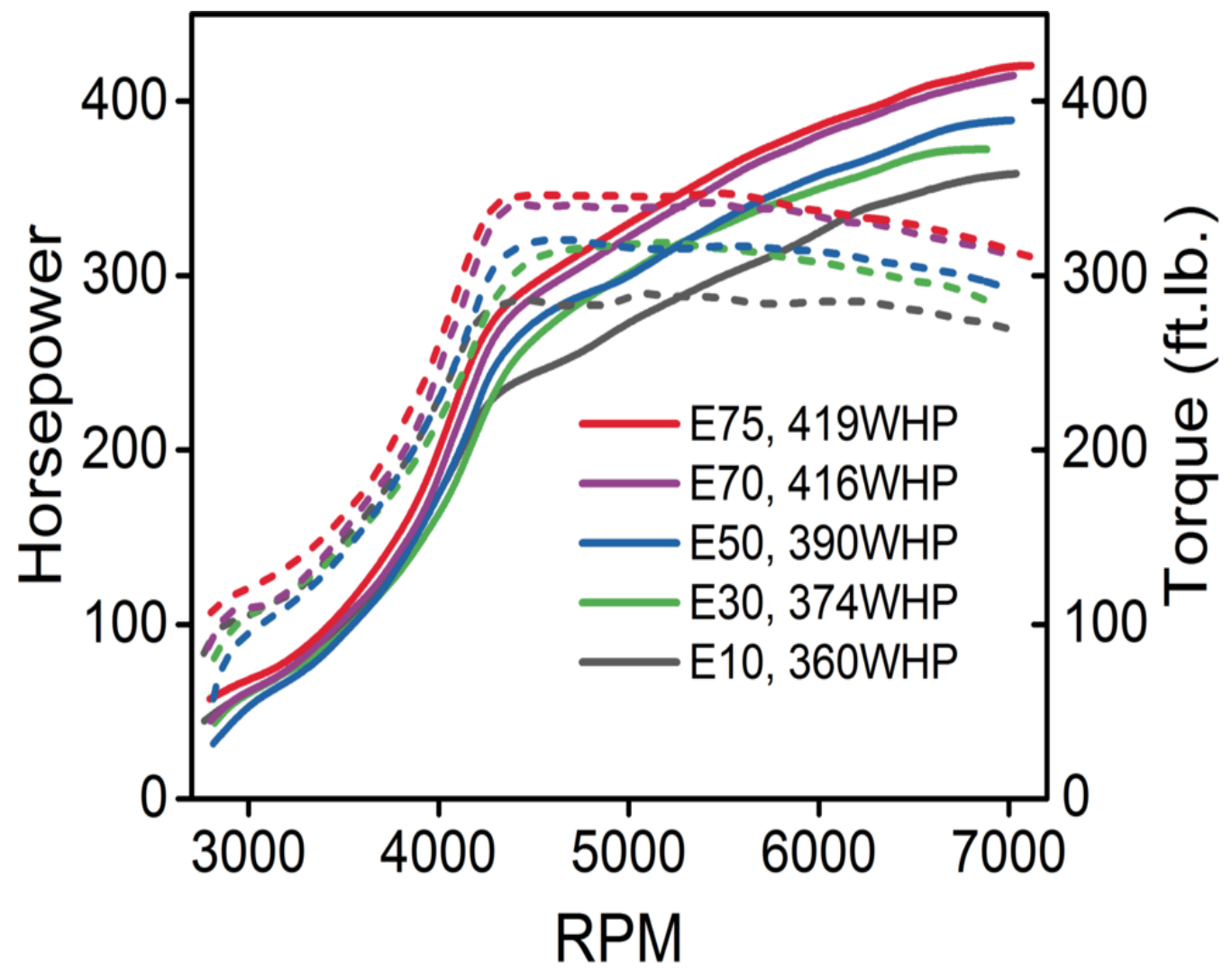

Fontes: [3], [4] 


\section{Fator $e_{\zeta}(\zeta, Y)$}

O fator $e_{\zeta}(\zeta, \Upsilon)$ procura computar as perdas na eficiência por desvio do ângulo ótimo de ignição. Para $\Upsilon=0$, temos [2, adaptado]:

$$
e_{\zeta}(\zeta, 0)=1-k_{\zeta}(0) \cdot\left(\zeta-\zeta_{0}\left(\omega_{e}, p_{a d m}, 0\right)\right)^{2}
$$

Aonde,

$k_{\zeta}(0)$ : Constante; $\zeta_{0}$ : Ângulo ótimo de ignição; $p_{a d m}$ : Pressão no coletor de admissão no centro de admissão;

Ao levantar estas curvas para diferentes composições, observa-se que a seguinte identificação pode ser realizada para composição variada:

$$
e_{\zeta}(\zeta, \Upsilon)=1-k_{\zeta}(\Upsilon) \cdot\left(\zeta-\zeta_{0}\left(\omega_{e}, p_{a d m}, \Upsilon\right)\right)^{2}
$$

com,

$$
k_{\zeta}(\Upsilon)=a \cdot \Upsilon^{2}+b \cdot \Upsilon+c
$$

Como a velocidade turbulenta de chama influi em como a eficiência térmica varia conforme ocorre excursões do ângulo de ignição fora da posição ótima, e sendo a velocidade turbulenta de chama dependente da velocidade do motor [5], pode-se fazer também:

$$
k_{\zeta}(\Upsilon)=\left(a \cdot \Upsilon^{2}+b \cdot \Upsilon+\mathrm{c}\right) \cdot\left(1+\mathrm{d} \cdot \omega_{e}\right)
$$

Aonde $a, b, c$ e $d$ são constantes.

De forma ao leitor entender por que consideramos $k_{\zeta}(Y)$ como uma função quadrática, reproduzimos na figura 2 um levantamento de torque para diferentes composições, baseando-se no mesmo método da seção 1, procurando obter uma 
visão qualitativa de como a eficiência varia com o ângulo de ignição conhecendo como que o torque varia com o ângulo de ignição, conforme equação abaixo:

$$
\frac{\partial T_{e}}{\partial \zeta} \approx K_{3} \cdot \frac{\partial e_{\zeta}(\zeta, \Upsilon)}{\partial \zeta}
$$

Repare como na figura 2 a abertura das parábolas, conforme aumenta-se a concentração de etanol, vai aumentando, atinge um valor máximo, e após vai diminuindo. Por isso que a função $k_{\zeta}(\Upsilon)$ foi aproximada como uma função quadrática.

Figura 2: Curvas $T_{e}=f(\zeta)$ para diferentes composições EX. Para o levantamento, usou-se rotação 5000RPM, posição da borboleta 26\% e $\lambda=1$.

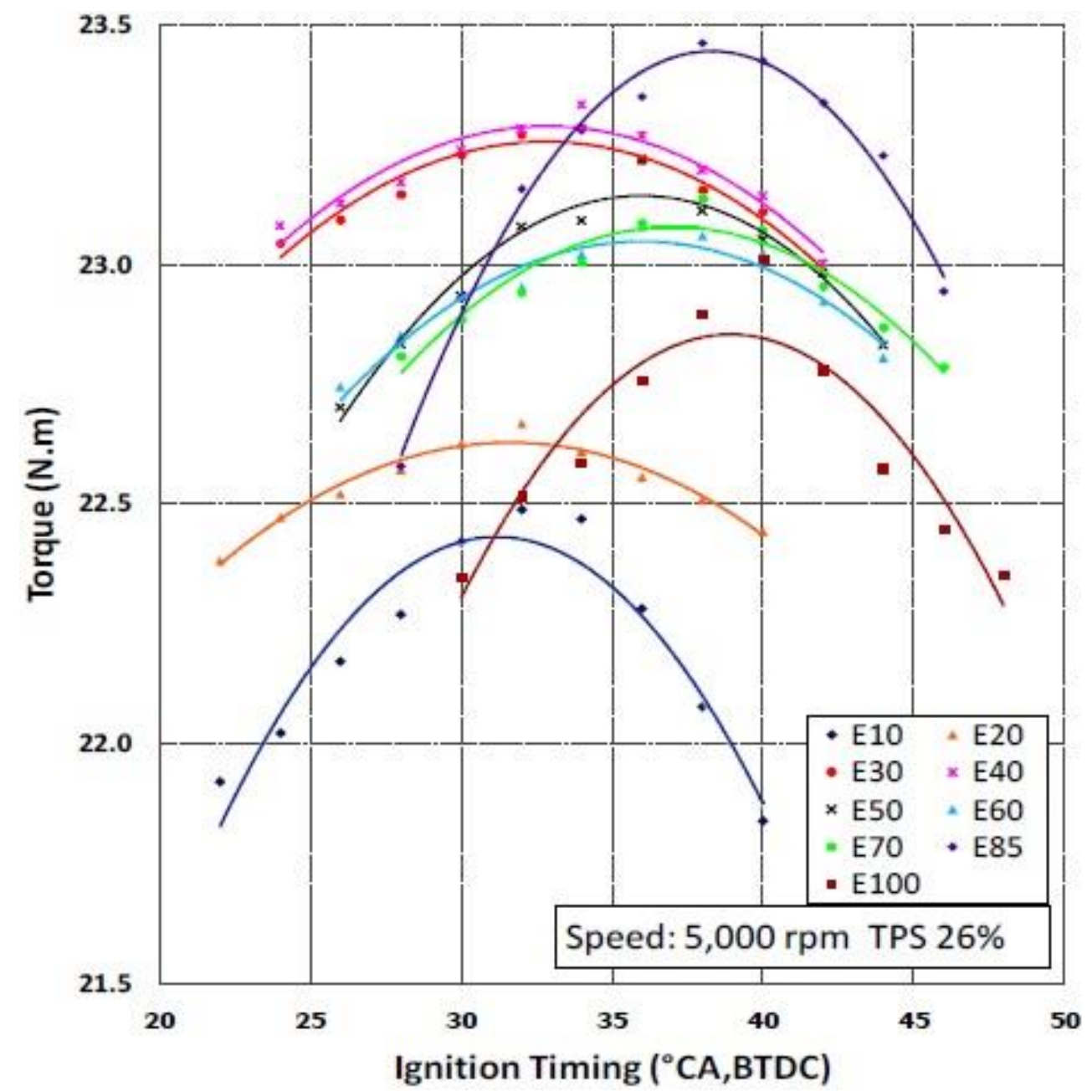

Fonte: [6] 


\section{Fator $e_{e g r}\left(x_{e g r}\right)$}

Como o acréscimo de diluentes na mistura de ar admitido através de recirculação provoca excursões no ângulo ótimo de ignição, segundo uma relação linear $\left(K_{1}\right.$ constante) [7], podemos fazer, aglutinando os fatores $e_{\text {egr }}\left(x_{\text {egr }}\right)$ e $e_{\zeta}(\zeta, 0)$ :

$$
e_{\zeta, \text { egr }}\left(\zeta, x_{e g r}, \Upsilon\right)=1-k_{\zeta}(\Upsilon) \cdot\left(\zeta-\zeta_{0}\left(\omega_{e}, p_{a d m}, \Upsilon\right)-K_{1} \cdot x_{e g r}\right)^{2}
$$

Repare que estamos desconsiderando uma relação entre a constante $K_{1}$ e a composição variada, pois a forma como a taxa de diluentes influi na velocidade laminar de chama não depende da composição variada [5].

Porém, caso se prefira estudar os dois fatores em separado, pode-se fazer conforme equação (13) [2]. Repare que desprezamos a influência da composição variada neste fator conforme discutido acima.

$$
e_{e g r}\left(x_{e g r}\right)=1-k_{e g r, 1} \cdot\left(1+k_{e g r, 2} \cdot \omega_{e}\right) \cdot x_{e g r}^{2}
$$

Repare que existe uma sensibilidade quadrática, ponderada pela constante $k_{e g r, 1}$, da eficiência com relação à taxa de recirculação, conforme também mostrado na equação (12). O fator $\left(1+k_{e g r, 2} \cdot \omega_{e}\right)$ busca computar o já discutido efeito da velocidade do motor na velocidade turbulenta de chama.

\section{Fator $e_{\lambda}(\lambda, Y)$}

O fator $e_{\lambda}(\lambda, \Upsilon)$ procura incluir na modelagem perdas devido ao déficit e excesso de oxigênio. Outros fenômenos a serem considerados são a combustão incompleta, que em termos energéticos pode ser desprezada [2][8], e a reação de mudança do vapor de água (RMV), que é mais significativa energeticamente, e pode influenciar a eficiência térmica de forma diferente para cada composição de combustível devido à Lei de Le Chatelier. 
Podemos formular o fator $e_{\lambda}(\lambda, \Upsilon)$ como [2]:

$$
e_{\lambda}(\lambda, 0)=\left\{\begin{array}{lr}
\gamma_{1} \cdot \lambda-\gamma_{0} & \text { para } \lambda_{\text {min }}<\lambda<1 \\
e_{\lambda, 1}+\left(1-e_{\lambda, 1}\right) \cdot \sin \frac{\lambda-\lambda_{1}}{1-\lambda_{1}} & \text { para } \lambda_{1}<\lambda<\lambda_{2} \\
1 & \text { para } \lambda_{2}<\lambda<\lambda_{\text {max }}
\end{array}\right.
$$

Aonde,

$e_{\lambda, 1}=\gamma_{1} \cdot \lambda-\gamma_{0}(15) ; \lambda_{1}=0.95$ (sugestão); $\lambda_{2}=1.0285$ (sugestão); $\gamma_{1}, \gamma_{0}$ : Parâmetros a serem identificados;

Deve-se ter, por razões de continuidade, $\lambda_{2}=\lambda_{1}+\frac{\pi}{2} \cdot\left(1-\lambda_{1}\right)(16)$, e deve-se adotar os limites $\lambda_{\min }$ e $\lambda_{\max }$ nos quais ocorre falhas de ignição por excesso e falta de combustível, respectivamente.

Uma sugestão é identificar $\gamma_{1}$ e $\gamma_{0}$ como funções afim na composição variada. Para o leitor ter visão do formato das curvas, segue figura 3 abaixo. Porém, note que este levantamento se refere apenas à eficiência da combustão, ou seja, às perdas nos gases de escape, que de fato é o fenômeno que mais diferencia as perdas energéticas no contexto de composição variada.

Figura 3: Eficiência da combustão em função da razão equivalente combustível/ar para composição variada. ULG é gasolina E0 regular sem chumbo.

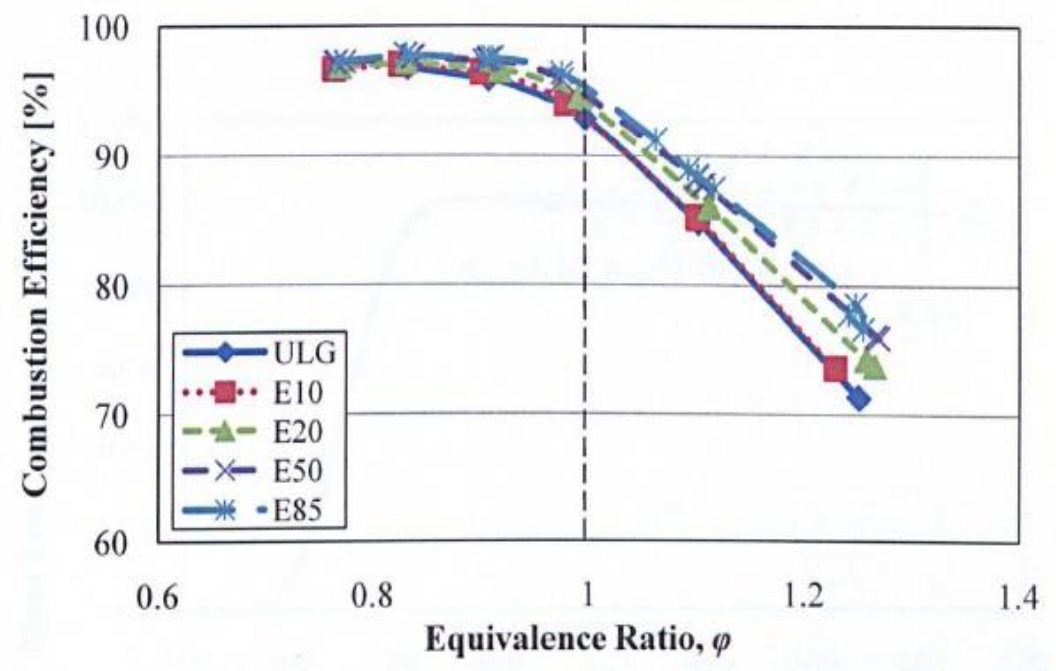

Fonte: [9] 


\section{Fatores $\boldsymbol{p}_{\text {meof }}($.$) e \boldsymbol{p}_{\text {meog }}(0)$}

Estes dois fatores constituem as perdas por atrito e funcionamento de dispositivos auxiliares, conforme discutido. Adotando $p_{m e 0}($.$) como o somatório destas$ perdas, temos que [2]:

$$
\begin{gathered}
p_{\text {me0 }}(.)=p_{\text {meog }}(0)+p_{\text {meof }}(.) \\
p_{\text {me0 }}(.)=p_{\text {meog }}(0)+k_{1}\left(\vartheta_{e}\right) \cdot\left(k_{2}+k_{3} \cdot S^{2} \cdot \omega_{e}^{2}\right) \cdot \Pi_{e, \max } \cdot \sqrt{\frac{k_{4}}{B}}
\end{gathered}
$$

Aonde,

$k_{1}\left(\vartheta_{\infty}\right)=1.44 \cdot 10^{5}(\mathrm{~Pa}) ; k_{2}=0.46 ; k_{3}=9.1 \cdot 10^{-4}\left(\mathrm{~s}^{2} \cdot \mathrm{m}^{-2}\right) ; k_{4}=0.075(\mathrm{~m}) ; S$ : Curso do pistão; B: Diâmetro do pistão; $\vartheta_{e}$ : Temperatura do motor; $\vartheta_{\infty}$ : Temperatura do motor quando este está plenamente aquecido; $\Pi_{e, \max }$ : a razão máxima de pressão no compressor. Para motores naturalmente aspirados considere que $\Pi_{e, \max }=1$ [10]. Abaixo, o equacionamento de $k_{1}\left(\vartheta_{\infty}\right)$, conforme [11].

$$
\begin{gathered}
k_{1}\left(\vartheta_{e}\right)=1-0.006 \cdot\left(\vartheta_{\text {eng,m }}-\vartheta_{\text {eng,m0 }}\right) \\
-2.25 \cdot 10^{-6} \cdot\left(\vartheta_{\text {eng,m }}-\vartheta_{\text {eng,m0 }}\right)^{3}-0.06 \cdot \vartheta_{e b}-\vartheta_{e b 0} \\
-2.25 \cdot 10^{-6} \cdot \vartheta_{e b}-\vartheta_{\text {eb0 }}{ }^{3}+1 \\
\vartheta_{\text {eng,m }}=\frac{\vartheta_{\text {eng,out }}+\vartheta_{\text {eng,in }}}{2}
\end{gathered}
$$

Aonde,

$\vartheta_{\text {eng,out }}:$ Temperatura do líquido refrigerador saindo do motor; $\vartheta_{\text {eng,in }}$ : Temperatura do líquido refrigerador entrando no motor; $\vartheta_{\text {eng,m0 }}: \vartheta_{\text {eng,m }}$ quando o motor está plenamente aquecido; $\vartheta_{e b}$ : Temperatura na carcaça do motor (do inglês: engine block); $\vartheta_{e b 0}: \vartheta_{e b}$ quando o motor está plenamente aquecido. Para a identificação destas temperaturas, consultar referências de modelagem do sistema térmico do motor, como a referência [11]. 
De forma a poder identificar o valor de $p_{m e 0}($.$) para diferentes velocidades \mathrm{e}$ temperatura do motor, para então poder identificar $k_{1}\left(\vartheta_{e}\right)$ e $p_{\text {meog }}(0)$, recomenda-se fazer a curva de Williams para diferentes situações. Como a curva de Williams deve ser traçada sem mudanças de condições que propiciem mudanças significativas de eficiência, recomenda-se o uso de taxa de recirculação ótima, razão ar/combustível próxima da estequiometria e ângulo ótimo de ignição e, ao procurar traçar a curva de Williams para determinada velocidade do motor, buscar conseguir pontos em velocidades pelo menos que sejam próximas a desejada, caso se tenha dificuldades em manipular tal velocidade. Para mais informações sobre curva de Williams, consultar [2] ou [12].

As influências da composição variada no atrito de forma geral e na energia perdida no funcionamento dos dispositivos auxiliares são desprezíveis, portanto não se deve levar em consideração a composição variada nesta identificação.

\section{CONCLUSÃO}

Neste artigo, expomos a formulação matemática da modelagem da eficiência térmica e do torque efetivo. A seguir apresentamos cada elemento das formulações e como identificá-los. Desta forma, além de possibilitar ao leitor estratégias mais elaboradas de controle, pois agora dispõe-se de um modelo, procurou-se mostrar quais os fatores que mais influem no torque efetivo e de que forma exercem esta influência.

\section{REFERÊNCIAS}

[1] MELO, T. de; MACHADO, G.; CARVALHO, L. de Oliveira; BELCHIOR, C. et al. In Cylinder Pressure Curve and Combustion Parameters Variability with Ethanol Addition. SAE Technical Paper. 2012.

[2] GUZZELLA, Lino; ONDER, Christopher. Introduction to modeling and control of internal combustion engine systems. 2. ed. Springer Science \& Business Media, 2010. 
[3] SCHMUCKER, Abrin. \#OneLapGSR // Part 7: The Long Road (to Michigan). Disponivel em: <http://www.tracktuned.com/feed/2017/2/19/onelapgsr-part-7-thelong-road>. Acesso em: 28 de abril de 2017.

[4] SCHMUCKER, Abrin. 2013 Evo X with CBRD RBX EFR7163 kit on the dyno. Disponível em: < https://www.youtube.com/watch?v=EjtP1-scZnA>. Acesso em: 28 de abril de 2017.

[5] YELIANA. Parametric Combustion Modeling for Ethanol-Gasoline Fuelled Spark Ignition Engines. 2010. Tese de Doutorado. Michigan Technological University.

[6] PHUANGWONGTRAKUL, S.; WANNATONG, K.; LAUNGNARUTAI T.; WECHSATOL, W.; Suitable Ignition Timing and Fuel Injection Duration for EthanolGasoline Blended Fuels in a Spark Ignition Internal Combustion Engine; Proc. of the Intl. Conf. on Future Trends in Structural, Civil, Environmental and Mechanical Engineering; FTSCEM 2013.

[7] MOSKWA, John Joseph. Automotive engine modeling for real time control. 1988. Tese de Doutorado. Massachusetts Institute of Technology.

[8] NÜESCH, Sandro Patrick. Analysis and control of multimode combustion switching sequence. 2015. Tese de Doutorado. The University of Michigan.

[9] ALRAYYES, Taleb. The effect of ethanol-gasoline blends on SI engine energy balance and heat transfer characteristics. 2011. Tese de Doutorado. University of Nottingham.

[10] ERIKSSON, Lars; NIELSEN, Lars. Modeling and control of engines and drivelines. John Wiley \& Sons, 2014.

[11] KANNE, Elena Cortona. Engine thermomanagement for fuel consumption reduction. 2000. Tese de Doutorado. ETH Zurich. 
[12] BRUNETTI, Franco; GARCIA, Oswaldo. Motores de combustão interna. São Paulo: Blucher. v.1, 2012. 